\title{
Complete elimination of nonlinear light-matter interactions with broadband ultrafast laser pulses
}

\author{
Chuan-Cun Shu, ${ }^{1,2, *}$ Daoyi Dong, ${ }^{1,3}$ Ian R. Petersen, ${ }^{1}$ and Niels E. Henriksen ${ }^{2, \dagger}$ \\ ${ }^{1}$ School of Engineering and Information Technology, University of New South Wales, Canberra, ACT 2600, Australia \\ ${ }^{2}$ Department of Chemistry, Technical University of Denmark, Building 207, DK-2800 Kongens Lyngby, Denmark \\ ${ }^{3}$ Department of Chemistry, Princeton University, Princeton, New Jersey 08544, USA
}

(Received 9 September 2016; published 10 March 2017)

\begin{abstract}
The absorption of a single photon that excites a quantum system from a low to a high energy level is an elementary process of light-matter interaction, and a route towards realizing pure single-photon absorption has both fundamental and practical implications in quantum technology. Due to nonlinear optical effects, however, the probability of pure single-photon absorption is usually very low, which is particularly pertinent in the case of strong ultrafast laser pulses with broad bandwidth. Here we demonstrate theoretically a counterintuitive coherent single-photon absorption scheme by eliminating nonlinear interactions of ultrafast laser pulses with quantum systems. That is, a completely linear response of the system with respect to the spectral energy density of the incident light at the transition frequency can be obtained for all transition probabilities between 0 and $100 \%$ in multilevel quantum systems. To that end, a multiobjective optimization algorithm is developed to find an optimal spectral phase of an ultrafast laser pulse, which is capable of eliminating all possible nonlinear optical responses while maximizing the probability of single-photon absorption between quantum states. This work not only deepens our understanding of light-matter interactions, but also offers a way to study photophysical and photochemical processes in the "absence" of nonlinear optical effects.
\end{abstract}

DOI: 10.1103/PhysRevA.95.033809

Exploring the interaction of light with matter (i.e., atoms and molecules) at the ultimate limit of single photons is a topic of much current interest in many disciplines of science. This includes topics such as generating single photon sources [1-3], storing single photons in quantum memory devices [4,5], and controlling the interactions between single photons and matter [6-8]. When a beam of light interacts with matter with quantized energy levels, optical absorption and emission are fundamental processes corresponding to a transition from one energy level to another. The rate of absorption has a component proportional to the energy density of the beam. The transition rate also contains terms of higher order, i.e., nonlinear terms in the energy density. The linear term in the absorption rate corresponds to the excitation in which a single photon is absorbed, whereas the nonlinear terms correspond to the excitation in which two or more photons are absorbed [9].

The probability of pure single-photon absorption under normal circumstances is very low. One of the major difficulties in realizing single-photon absorption with unit probability is to overcome decoherence (e.g., population relaxation) due to the intrinsic fragility of quantum states, which is also a common challenge for quantum technology. Ultrafast laser pulses provide an alternative approach to manipulate many quantum processes on extremely short time scales (attoto picoseconds) before decoherence plays a role [10-13]. When such a laser pulse that contains a huge number of photons within the broad bandwidth excites matter, another major difficulty due to nonlinear optical effects may emerge. Based on a mature spectral-phase-shaping technique [14], considerable theoretical and experimental effort has been directed toward the study of single-photon phase control in the weak-field regime [15-20]. Related theoretical work has

\footnotetext{
*c.shu@unsw.edu.au

${ }^{\dagger}$ neh@kemi.dtu.dk
}

shown that a nearly linear response of the system as a function of laser energy density is possible, somewhat beyond the strictly weak-field limit, provided appropriate laser spectralphase modulation is introduced [21]. Furthermore, seminal work by Silberberg et al. [10,22,23] has demonstrated how this approach can be used in the modulation of multi (two)-photon transitions in atoms, and a direct signature has been observed in which a two-photon nonlinear optical process in molecules can be significantly affected by chirping the spectral phase of the laser pulse at very low light intensities [24]. Inspired by these previous studies, a fundamentally important but largely unexplored question is whether pure spectral-phase shaping of an ultrafast laser pulse can lead to complete elimination of nonlinear optical effects.

In this article, we demonstrate that a completely linear absorption probability, as a function of the energy density at the transition frequency, can be obtained for all transition probabilities between the minimum 0 and the maximum $100 \%$ in a prototypical multilevel quantum system. To that end, we develop a monotonically convergent multiobjective optimization algorithm, which combined with a perturbation theory analysis is utilized to find the optimal spectral phase of a laser pulse for minimizing nonlinear optical effects while maximizing the probability of single-photon transition. The robustness of the maximal single-photon absorption against the influence of spectral field noise is also examined.

The basic aim of this work is sketched in Fig. 1. We consider the simplest multilevel quantum-mechanical system (atom or molecule), consisting of two lower states $|g\rangle$ and $|s\rangle$ and an excited state $|f\rangle$ with eigenenergies $E_{g}<E_{s}<E_{f}$. The transitions between the lower states $|g\rangle,|s\rangle$ and the excited states $|f\rangle$ are dipole allowed, but the transition between the states $|g\rangle$ and $|s\rangle$ is dipole forbidden. An ultrafast laser pulse is used to excite such a quantum system, whose lifetimes in the states $|s\rangle$ and $|f\rangle$ are assumed to be much longer than the duration of the laser pulse. Such a multilevel 


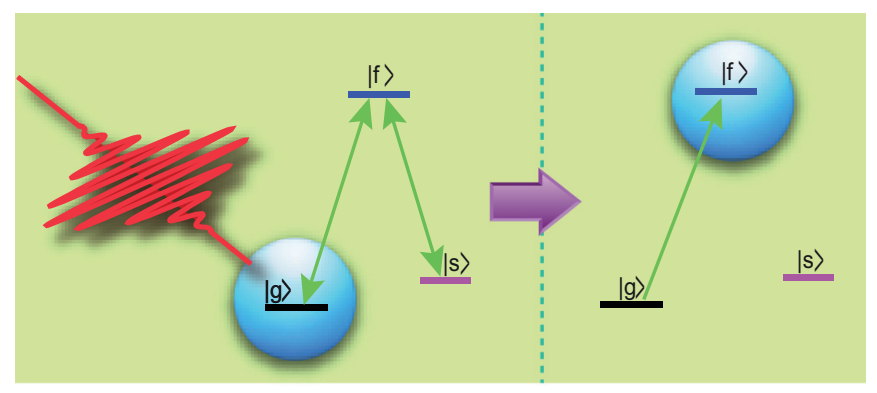

FIG. 1. The interaction of a laser field with a three-level ( $\Lambda$-type) quantum system consisting of two lower levels $|g\rangle$ and $|s\rangle$ and an upper level $|f\rangle$. A broadband ultrafast laser pulse is shaped to obtain a linear response of the population in the state $|f\rangle$ with respect to the energy density while eliminating all possible transitions to state $|s\rangle$.

model has been used for modeling a variety of optical schemes, including electromagnetically induced transparency (EIT) and photon storage [25-27] and stimulated Raman adiabatic passage (STIRAP) [28-30], where nonlinear multi (two)-photon transitions to the state $|s\rangle$ are taken advantage of in cancellation of population in the state $|f\rangle$. In contrast with these schemes, the present work aims to protect the absorption from $|g\rangle$ to $|f\rangle$ via destructive quantum interferences with multiphoton transition pathways.

The total Hamiltonian operator $\hat{H}(t)$ of the quantum system in interaction with a light field $\mathcal{E}(t)$ can be described by $\hat{H}(t)=\hat{H}_{0}-\hat{\mu} \mathcal{E}(t)$, where $\hat{H}_{0}$ is the field-free Hamiltonian operator and $\hat{\mu}$ the dipole operator. The wave function $|\Psi(t)\rangle$ of the quantum system, initially in the ground state $|g\rangle$, can be iteratively expanded to arbitrary order as $|\Psi(t)\rangle=\sum_{k=0}^{\infty}\left|\psi^{(k)}(t)\right\rangle$ with $\left|\psi^{(0)}(t)\right\rangle=|g\rangle$ and $\left|\psi^{(k+1)}(t)\right\rangle=-i \int_{-\infty}^{t} d t^{\prime} \mathcal{E}\left(t^{\prime}\right) \hat{\mu}_{I}\left(t^{\prime}\right)\left|\psi^{(k)}\left(t^{\prime}\right)\right\rangle$, where $\hat{\mu}_{I}(t)=$ $\exp \left(i \hat{H}_{0} t\right) \hat{\mu} \exp \left(-i \hat{H}_{0} t\right)$ is the dipole operator in the interaction picture. The electric field of the laser pulse can be expressed as

$$
\mathcal{E}(t)=\frac{1}{2 \pi} \operatorname{Re}\left[\int_{0}^{\infty} \boldsymbol{E}(\omega) \exp (-i \omega t) d \omega\right],
$$

with the complex-valued spectral field $\boldsymbol{E}(\omega)=$ $A(\omega) \exp [i \phi(\omega)]$ in terms of the real-valued spectral amplitude $A(\omega) \geqslant 0$ and the real-valued spectral phase $\phi(\omega)$. The energy of such a pulse can be expressed as $\mathbb{E}_{p} \propto \int_{-\infty}^{\infty} \mathcal{E}^{2}(t) d t \propto \int_{0}^{\infty} A^{2}(\omega) d \omega$, which is independent of the spectral phase $\phi(\omega)$. To first order, the transition probability from the ground state $|g\rangle$ to the final state $|f\rangle$, corresponding to single-photon absorption, is given by

$$
P_{f}^{(1)}=\left|\left\langle f \mid \psi^{(1)}(\infty)\right\rangle\right|^{2}=\mu_{f g}^{2} A^{2}\left(\omega_{f g}\right),
$$

where $\mu_{f g}=\langle f|\mu| g\rangle$ is the transition dipole moment and $\omega_{f g}=E_{f}-E_{g}$ is the transition frequency between the states $|g\rangle$ and $|f\rangle$. Thus, the probability of absorption depends linearly on the square of the spectral amplitude $A^{2}(\omega)$ at the resonant transition frequency $\omega_{f g}$, i.e., the spectral energy density, but is independent of the spectral phase. Furthermore, beyond first order in the interaction, odd-order perturbation terms will contribute to the transition probability to the state $|f\rangle$ and even-order terms will transfer population to the states $|s\rangle$ and $|g\rangle$, and a dependence on the spectral phase $\phi(\omega)$ is observed. The present work will show a coherent control scheme to realize an interesting limit of linear absorption from $|g\rangle$ to $|f\rangle$ by modulating the spectral phase of a single ultrashort pulse, where the effects of all higher-order perturbation terms are eliminated.

Solutions to analytically unaccessible maximization/minimization problems under consideration can be established in the framework of quantum optimal control theory (QOCT) [31-36]. Due to the technical complexity involved in acquiring either monotonic convergence or general applicability of the algorithms, however, the present problem is a challenge to the previously developed QOCT methods. We develop here a gradient-based multiobjective optimization algorithm that not only is capable of optimizing the spectral phase of the laser pulse in the frequency domain, but also ensures monotonic convergence to each control objective simultaneously. To formulate this method in an elegant mathematical form, a dummy variable $x \geqslant 0$ is employed to parametrize the spectral phase $\phi(\omega)$ with $\phi(x, \omega)$. As $x$ increases, the change of the final population $P_{\ell}=|\langle\ell \mid \Psi(\infty)\rangle|^{2}$ in an arbitrary quantum state $|\ell\rangle$ of the system can be written using the chain rule as

$$
\frac{d P_{\ell}}{d x}=\int_{0}^{\infty} \frac{\delta P_{\ell}}{\delta \phi(x, \omega)} \frac{\partial \phi(x, \omega)}{\partial x} d \omega .
$$

The spectral phase is updated from $\phi(x, \omega)$ to $\phi(x+d x, \omega)$ with

$$
\frac{\partial \phi(x, \omega)}{\partial x}=\int_{0}^{\infty} S\left(\omega^{\prime}-\omega\right) \sum_{\ell, \ell^{\prime}=1}^{M} k_{\ell}(x)\left[\Gamma^{-1}\right]_{\ell \ell^{\prime}} \frac{\delta P_{\ell^{\prime}}}{\delta \phi\left(x, \omega^{\prime}\right)} d \omega^{\prime},
$$

where the convolution function $S\left(\omega^{\prime}-\omega\right)$ is a filter for smoothing the updated spectral phase, and $\Gamma$ is a symmetric matrix composed of the elements $\Gamma_{\ell \ell^{\prime}}=\int_{0}^{\infty} \delta P_{\ell} / \delta \phi(x, \omega) \int_{0}^{\infty} S\left(\omega^{\prime}-\right.$ $\omega) \delta P_{\ell^{\prime}} / \delta \phi\left(x, \omega^{\prime}\right) d \omega^{\prime} d \omega$. By inserting Eq. (4) into Eq. (3) (see details in Appendix), we can verify that $P_{\ell}$ can be monotonically increased (decreased) simultaneously with $k_{\ell}>0$ $\left(k_{\ell}<0\right)$. Note that the optimization algorithm indicated in Eq. (4) is independent of the dimension of Hamiltonian, ensuring its applicability to complex multilevel quantum systems. To perform this algorithm, the quantum system is driven with an initial guess of the spectral phase $\phi\left(x_{0}, \omega\right)=0$ associated with the temporal field $\mathcal{E}\left(x_{0}, t\right)$, and the generated wave function $\Psi(t)$ is used to calculate the gradients of $P_{\ell}$ with respect to the spectral phase $\phi\left(x_{0}, \omega\right)$ for getting the first gradient $\partial \phi\left(x_{0}, \omega\right) / \partial x$ (see details in Appendix). Equation (4) is solved (e.g., by using the Euler method) to obtain a new spectral phase $\phi\left(x_{1}=x_{0}+d x, \omega\right)$ and the corresponding time-dependent laser field $\mathcal{E}\left(x_{1}, t\right)$ is calculated according to Eq. (1). The spectral phase is iteratively updated from $\phi\left(x_{1}, \omega\right)$ to $\phi\left(x_{2}=x_{1}+d x, \omega\right), \ldots, \phi\left(x_{n}, \omega\right)$ until $P_{\ell}$ converges to the desired control objectives.

To eliminate the nonlinear light-matter interaction terms, we employ this optimization algorithm to optimize $P_{f}$ to be as close to the linear absorption probability $P_{f}^{(1)}$ as possible while minimizing $P_{s}$ in the state $|s\rangle$. The unshaped laser field $\mathcal{E}\left(x_{0}, t\right)$ is taken to be an experimentally accessible 

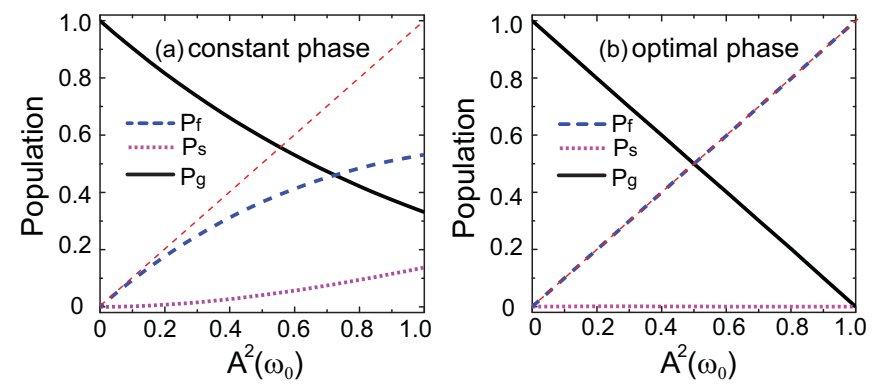

FIG. 2. The final populations in the three states with (a) constant spectral phase and (b) optimal spectral phases with respect to $A^{2}\left(\omega_{0}\right)$, proportional to the pulse energy. The straight line shows the linear scaling of the transition probability $P_{f}$ to the upper level, which is valid at low transition probabilities with a constant laser phase and for all transition probabilities with an optimized phase.

Gaussian transform-limited pulse with the center frequency of $\omega_{0}=12500 \mathrm{~cm}^{-1}(800 \mathrm{~nm})$ and the FWHM of $30 \mathrm{fs}$. The eigenenergies of the three-level quantum system are chosen as $E_{g}=0, E_{s}=0.02 \omega_{0}, E_{f}=\omega_{0}$, and the transition dipole moments between the two lower states and the excited state are set to $\mu_{g f}=\mu_{s f}=1.0$ a.u. for convenience. A normalized Gaussian spectral filter $S\left(\omega^{\prime}-\omega\right)=\exp \left[-4 \ln 2\left(\omega^{\prime}-\omega\right)^{2} / \sigma^{2}\right]$ with a bandwidth of $\sigma=80 \mathrm{~cm}^{-1}$ is used in Eq. (4). Figure 2(a) shows the final populations in the three states as a function of $A^{2}\left(\omega_{0}\right)$ with constant $\phi(\omega)=0$ spectral phase. The linear optical transition to the state $|f\rangle$, observed in the weak-field limit regime, is significantly affected as the energy density increases, resulting in population transfer to the state $|s\rangle$.

As seen from Eq. (2), choosing the square of the (peak) spectral amplitude at the critical value of $A^{2}\left(\omega_{0}\right)=1.0$ corresponding to $P_{f}^{(1)}=1.0$ could, in the absence of nonlinear interactions, completely excite the quantum system from the ground state $|g\rangle$ to the final state $|f\rangle$. We first fix the spectral amplitude at $A\left(\omega_{0}\right)=1.0$ (corresponding to the peak intensity of $I_{0}=8.0544 \times 10^{10} \mathrm{~W} / \mathrm{cm}^{2}$ for the transform-limited pulse) and then use the optimization algorithm to maximize $P_{f}\left(k_{f}>\right.$ $0)$ while minimizing $P_{s}\left(k_{s}<0\right)$. Our results show that by iteratively optimizing the spectral phase, $P_{f}$ can be maximized to unity with high precision (see Fig. 6 in the Appendix, where $P_{f}>0.99999$ and $\left.P_{s}<1.0 \times 10^{-8}\right)$. Furthermore, by using this optimized spectral phase as the initial input, we further examine the final population responses of the three states with respect to the laser pulses with $A^{2}\left(\omega_{0}\right)<1.0$ (see Fig. 7 in Appendix). The nonlinear optical transitions to the intermediate state $|s\rangle$ can be greatly reduced to $P_{s}<5 \times 10^{-3}$, and the final population $P_{f}$ is always greater than $P_{f}^{(1)}$ for all of $A^{2}\left(\omega_{0}\right)<1.0$. This result provides an accessible approach to decrease $P_{f}\left(k_{f}<0\right)$ as close to $P_{f}^{(1)}$ as possible while further decreasing $P_{s}\left(k_{s}<0\right)$ by using the present optimization algorithm. Figure 2(b) shows the final optimized populations in the three states as a function of $A^{2}\left(\omega_{0}\right)$. A linear response of $P_{f}$ with respect to $A^{2}\left(\omega_{0}\right)$ is restored while efficiently suppressing nonlinear optical transitions to the state $|s\rangle$. As a result, a linear superposition $\alpha|g\rangle+\beta|f\rangle$ is obtained, where the coefficients $\alpha$ and $\beta$ are complex numbers satisfying $|\alpha|^{2}+|\beta|^{2}=1$. In the field of quantum computing, this superposition state corresponds to a qubit.

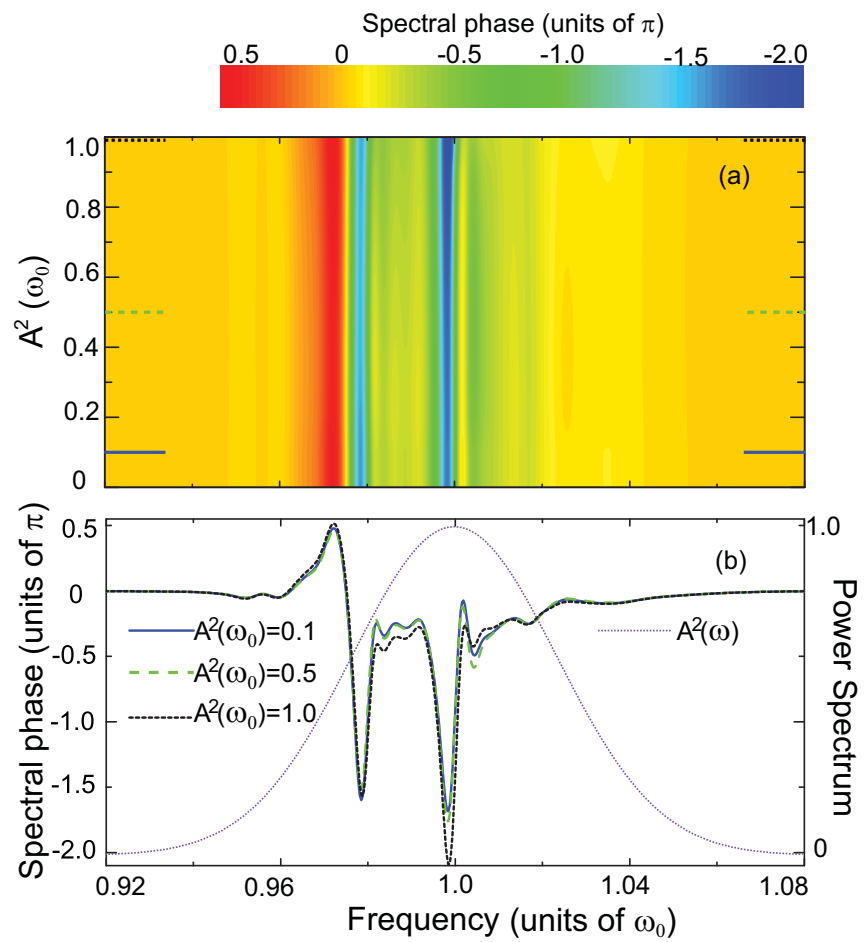

FIG. 3. The optimized spectral phases used in Fig. 2(b). (a) The optimized spectral phase as a function of $A^{2}\left(\omega_{0}\right)$. (b) The optimized spectral phases of the laser pulse with $A^{2}\left(\omega_{0}\right)=0.1$ (solid line), 0.5 (dashed line), and 1.0 (dashed line). The Gaussian curve shows [except for the overall scaling $A^{2}\left(\omega_{0}\right)$ ] the normalized power spectrum, i.e., the fixed spectral distribution of the laser pulses.

The optimized spectral phases at different values of $A^{2}\left(\omega_{0}\right) \leqslant 1.0$ are plotted in Fig. 3. It is observed that the optimized spectral phases are mainly modulated around the two fundamental frequencies $\omega_{0}$ and $\omega_{0}-\omega_{s g}=0.98 \omega_{0}$, leading to a substantial reduction of multiphoton (e.g., resonance Raman) transitions to the state $|s\rangle$. To gain insight into the effect of the optimized spectral phase on the dynamics of the final state, Fig. 4 shows a comparison of $P_{f}^{(1)}(t)$ and $P_{f}(t)$ with constant and optimized spectral phases at three different values of $A^{2}\left(\omega_{0}\right)$. For a constant spectral phase, the pulse smoothly transfers the population to the final state $|f\rangle$, where the differences between the first-order perturbation simulations and the exact solution to the Schrödinger equation imply that higher-order perturbations and therefore nonlinear optical effects play a role. The optimized spectral phases prolong the pulse durations from the femtosecond to the picosecond regime and almost restore the behavior of $P_{f}^{(1)}(t)$ under the first-order description, especially at lower intensities [see Fig. 4( $\left.\mathrm{a}^{\prime}\right)$ ], where the high-order perturbation terms are rather weak. It is noteworthy that first-order perturbation theory correctly predicts all post-pulse transition probabilities between 0 and 1. The slight differences of the transient dynamics between the first-order perturbation simulations and the exact calculations [see Figs. 4( $\left.b^{\prime}\right)$ and $4\left(c^{\prime}\right)$ ] can be attributed to the fact that the nonlinear optical transitions take place during the laser-system interactions, but their contributions to the final absorption probability to the state $|f\rangle$ are completely eliminated. Note that the transient dynamics induced by the spectral-phase 

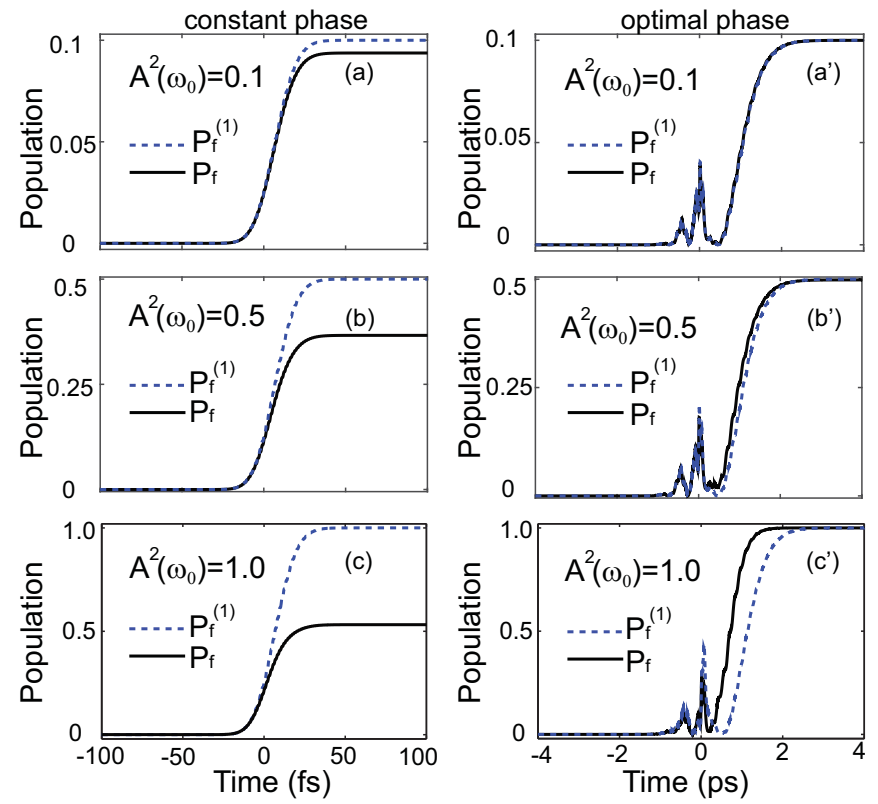

FIG. 4. A comparison of $P_{f}^{(1)}(t)$ (dashed line, obtained by firstorder perturbation calculation) and $P_{f}(t)$ (the exact time-dependent Schrödinger equation solution) with constant (left panels) and optimized (right panels) spectral-phase pulses at (a, $\left.\mathrm{a}^{\prime}\right) A^{2}\left(\omega_{0}\right)=0.1$, $\left(\mathrm{b}, \mathrm{b}^{\prime}\right) A^{2}\left(\omega_{0}\right)=0.5$, and $\left(\mathrm{c}, \mathrm{c}^{\prime}\right) A^{2}\left(\omega_{0}\right)=1.0$.

optimization exhibit strong oscillations in the excited-state population which differ from the case of constant spectral phase. Oscillatory dynamics of the excited-state population in the perturbative regime of the interaction have been observed experimentally by linearly chirping the spectral phase of a laser pulse [37].

We now examine the robustness of this scheme against the influence of the control field noise, which has been identified as one of the key requirements in practical applications of quantum technology [38,39]. Due to various external or internal perturbations of laser sources, the temporal laser fields in the laboratory can be subject to stochastic noise in either the time or frequency domain. As an example, Fig. 5 shows the final excited-state population variations versus the laser field fluctuations, where the fixed spectral amplitude $A(\omega)$ at $A^{2}\left(\omega_{0}\right)=1.0$ and the optimized spectral phase $\phi(\omega)$ are perturbed simultaneously with white Gaussian noise of $50 \approx 100 \mathrm{~dB}$ in signal-to-noise ratio (SNR). A high efficiency of the population transfer to the final state $|f\rangle$ can still be achieved with an admissible error lower than $10^{-4}$ when the SNR is over $70 \mathrm{~dB}$, which is possible using the current state-of-the-art laser techniques [40].

In summary, we have presented an optical phase modulation scheme for coherent light that can be utilized to completely eliminate nonlinear optical effects, leading to a linear absorption response from a low to a high energy level in a multilevel quantum system. The fundamental limit of single-photon absorption and therefore a linear superposition of two quantum states was achieved by transferring the optimal spectral phase of a broad-bandwidth ultrafast laser pulse onto the quantum wave function of the system. To that end, a versatile spectral-phase optimization algorithm was developed that can be used to monotonically approach

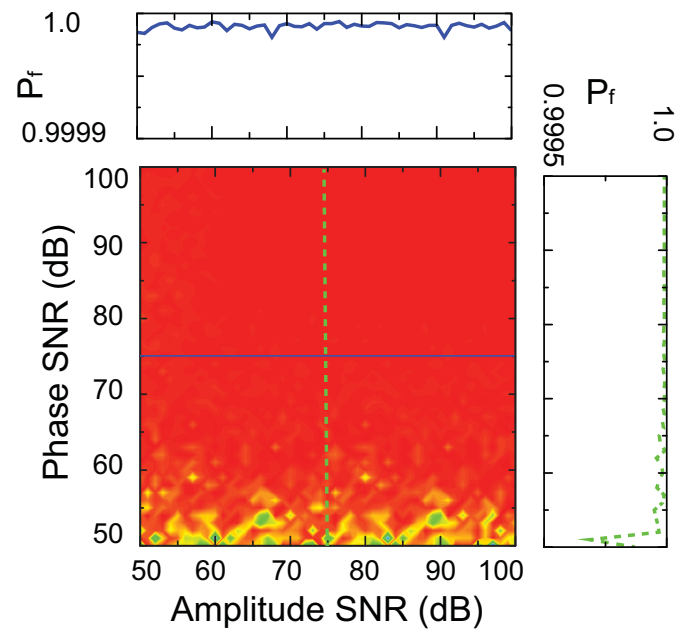

FIG. 5. The robustness of the perfect population transfer to the final state $|f\rangle\left(\right.$ at $\left.A^{2}\left(\omega_{0}\right)=1.0\right)$ against the influence of spectral field noise. In this simulation, a white Gaussian noise of $50 \approx 100 \mathrm{~dB}$ in signal-to-noise ratio (SNR) is added to the fixed spectral amplitude and the optimized spectral phase, and then the noised spectral field is transformed to obtain the temporal field of the laser pulse for driving the evolution of the quantum system.

multiple control objectives simultaneously. This single-photon absorption limit was found to be robust with tolerable influence of spectral field noise. These results suggest also an alternative approach to prepare a qubit in a multilevel quantum system. Since this multiobjective optimization algorithm is general for maximizing the probability of a single-photon transition while minimizing nonlinear optical transitions to multiple unwanted levels, the key idea introduced here could be extended to study more complex atoms and molecules as well as other quantum systems. This work can open a number of potential applications, including the manipulation of quantum wave functions and the extraction of a single photon from ultrafast laser pulses.

D.D. and I.R.P. acknowledge partial supports by the Australian Research Council under Grants No. DP130101658 and No. FL110100020. C.C.S. acknowledges the financial support by the Vice Chancellor's Postdoctoral Research Fellowship of University of New South Wales, Australia, and the hospitality provided by the Technical University of Denmark during his visit in February 2016.

\section{APPENDIX}

The total Hamiltonian operator $\hat{H}(t)$ of the quantum system in interaction with a light field $\mathcal{E}(t)$ can be described by $\hat{H}(t)=$ $\hat{H}_{0}-\hat{\mu} \mathcal{E}(t)$, where $\hat{H}_{0}$ is the field-free Hamiltonian operator and $\hat{\mu}$ the dipole operator. The time-dependent evolution of the quantum system is described by the wave function $\Psi(t)=\hat{U}(t,-\infty)|g\rangle$, where $\hat{U}(t,-\infty)$ is the corresponding unitary evolution operator governed by the time-dependent Schrödinger equation,

$$
i \frac{\partial \hat{U}(t,-\infty)}{\partial t}=\hat{H}(t) \hat{U}(t,-\infty), \quad \hat{U}(-\infty,-\infty) \equiv \mathbb{I},
$$


where $t \rightarrow-\infty$ denotes a time long before the pulse is turned on, whereas $t \rightarrow \infty$ corresponds to a time long after the pulse is turned off.

To formulate this optimization method, a dummy variable $x \geqslant 0$ is employed to parametrize the spectral phase $\phi(\omega)$ with $\phi(x, \omega)$. The gradient of the final population $P_{\ell}=$ $|\langle\ell|\hat{U}(\infty,-\infty)| g\rangle|^{2}$ in state $|\ell\rangle(\ell=g, s$ and $f)$ with respect to this dummy variable can be written using the chain rule as

$$
g_{\ell}(x) \equiv \frac{d P_{\ell}}{d x}=\int_{0}^{\infty} \frac{\delta P_{\ell}}{\delta \phi(x, \omega)} \frac{\partial \phi(x, \omega)}{\partial x} d \omega,
$$

where $g_{\ell}(x)>0$ corresponds to an increase in the final population in state $|\ell\rangle$, whereas $g_{\ell}(x)<0$ corresponds to decreasing the final population in $|\ell\rangle$. To optimize multiple objectives in a monotonic convergence fashion, the spectral phase can be updated from $\phi(x, \omega)$ to $\phi(x+d x, \omega)$ with

$$
\frac{\partial \phi(x, \omega)}{\partial x}=\int_{0}^{\infty} S\left(\omega^{\prime}-\omega\right) \sum_{\ell, \ell^{\prime}=1}^{M} k_{\ell}(x)\left[\Gamma^{-1}\right]_{\ell \ell^{\prime}} \frac{\delta P_{\ell^{\prime}}}{\delta \phi\left(x, \omega^{\prime}\right)} d \omega^{\prime},
$$

where the convolution function $S\left(\omega^{\prime}-\omega\right)$ is the filter for smoothing the updated spectral phase, and $\Gamma$ is a symmetric matrix composed of the elements $\Gamma_{\ell \ell^{\prime}}=\int_{0}^{\infty} \delta P_{\ell} /$ $\delta \phi(x, \omega) \int_{0}^{\infty} S\left(\omega^{\prime}-\omega\right) \delta P_{\ell^{\prime}} / \delta \phi\left(x, \omega^{\prime}\right) d \omega^{\prime} d \omega$. By inserting Eq. (A3) into Eq. (A2), we can verify that

$$
\begin{aligned}
g_{\ell}(x)= & \int_{0}^{\infty} c_{\ell}(x, \omega) \int_{0}^{\infty} S\left(\omega^{\prime}-\omega\right) \\
& \times \sum_{\ell^{\prime \prime}, \ell^{\prime}=1}^{M} k_{\ell^{\prime \prime}}(x)\left[\Gamma^{-1}\right]_{\ell^{\prime \prime} \ell^{\prime}} c_{\ell^{\prime}}\left(x, \omega^{\prime}\right) d \omega^{\prime} d \omega \\
= & \sum_{\ell^{\prime \prime}, \ell^{\prime}=1}^{M} k_{\ell^{\prime \prime}}(x)\left[\Gamma^{-1}\right]_{\ell^{\prime \prime} \ell^{\prime}} \Gamma_{\ell^{\prime} \ell} \\
= & \sum_{\ell^{\prime \prime}=1}^{M} k_{\ell^{\prime \prime}}(x) \delta_{\ell^{\prime \prime} \ell}
\end{aligned}
$$

is always greater than $\left(k_{\ell}>0\right)$ or less than $\left(k_{\ell}<0\right)$ zero, implying that all monotonic convergence conditions in Eq. (A2) are satisfied simultaneously. To perform this method, the gradients of $P_{\ell}$ with respect to the spectral phase $\phi(x, \omega)$ in Eq. (A2) are computed by

$$
\frac{\delta P_{\ell}}{\delta \phi(x, \omega)}=\int_{-\infty}^{\infty} \frac{\delta P_{\ell}}{\delta \mathcal{E}(x, t)} \frac{\partial \mathcal{E}(x, t)}{\partial \phi(x, \omega)} d t .
$$

where $\quad \partial \mathcal{E}(x, t) / \partial \phi(x, \omega)=-A(\omega) \sin [\phi(x, \omega)-\omega t] /(2 \pi)$. The gradients of $P_{\ell}$ with respect to the temporal laser field $\mathcal{E}(x, t)$ are calculated by

$$
\begin{aligned}
\frac{\delta P_{\ell}}{\delta \mathcal{E}(x, t)}= & -2 \operatorname{Im}\left\{\left\langle g\left|\hat{U}^{\dagger}(\infty,-\infty)\right| \ell\right\rangle\langle\ell| \hat{U}(\infty,-\infty)\right. \\
& \left.\times \hat{U}^{\dagger}(t,-\infty) \hat{\mu} \hat{U}(t,-\infty)|g\rangle\right\} .
\end{aligned}
$$

The spectral phase is updated according to the Euler method with $\phi(x+d x, \omega)=\phi(x, \omega)+d x[\partial \phi(x, \omega) / \partial x]$

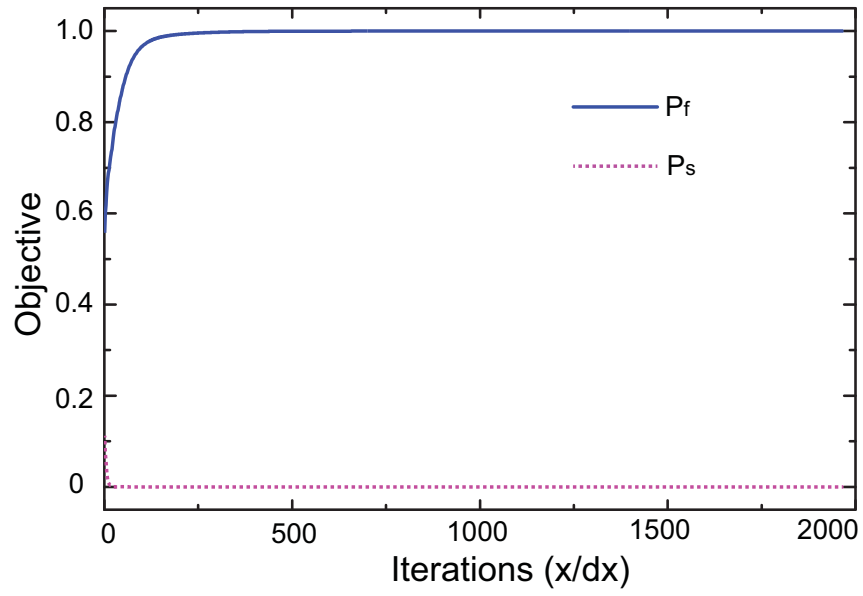

FIG. 6. The control objective as a function of iterations for realizing the fundamental limit of linear optical absorption at $A^{2}\left(\omega_{0}\right)=1$. The multiobjective optimization algorithm is used to maximize the final population on the state $|f\rangle$ while minimizing the final population on the state $|s\rangle$ in a monotonic convergence fashion.

using Eq. (A3). This phase is used to calculate the time-dependent laser field $\mathcal{E}(x+d x, t)$, which will increase $\left(k_{\ell}(x)>0\right)$ or decrease $\left(k_{\ell}(x)<0\right)$ the final population $P_{\ell}$ as compared with that by the laser field $\mathcal{E}(x, t)$. Note that this multiobjective optimization algorithm in principle can also be employed in the laboratory, where the gradients $\delta P_{\ell} / \delta \phi(x, \omega)$ in the frequency domain can be measured with stochastic sampling methods. Supplemental results are shown in Figs. 6 and 7 .

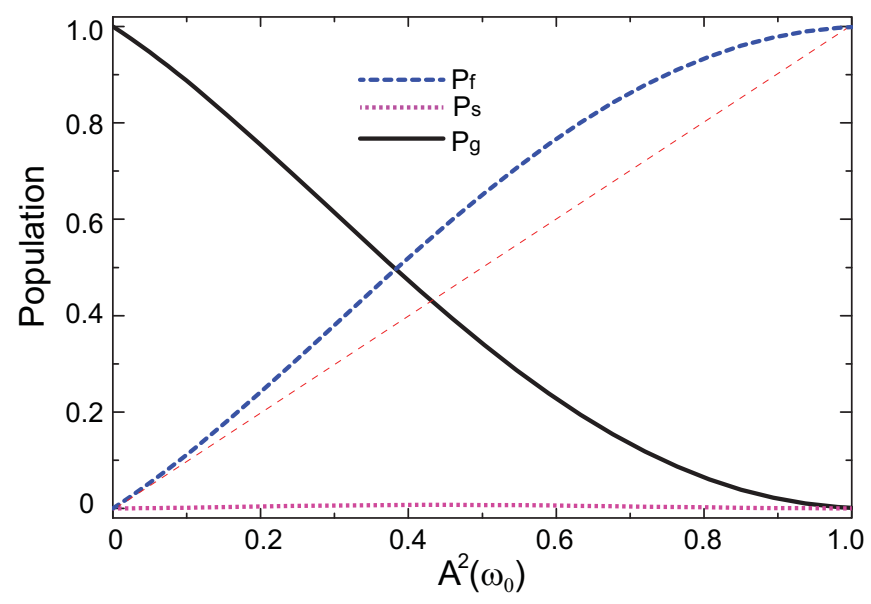

FIG. 7. The final populations in the three states as a function of the energy density $A^{2}(\omega)$ at the transition frequency $\omega_{0}$ with the optimized spectral phase obtained at $A^{2}\left(\omega_{0}\right)=1$. The nonlinear optical transitions to the intermediate state $|s\rangle$ are greatly reduced to $P_{s}<5 \times 10^{-3}$, and the final population $P_{f}$ is always greater than $P_{f}^{(1)}$ for all of $A^{2}\left(\omega_{0}\right)<1.0$. This result provides an accessible approach to decrease $P_{f}$ as close to $P_{f}^{(1)}$ as possible while further decreasing $P_{s}$ by using the presented multiobjective optimization algorithm. 
[1] S. Rosenblum, O. Bechler, I. Shomroni, Y. Lovsky, G. Guendelman, and B. Dayan, Nat. Photon. 10, 19 (2016).

[2] L. S. Costanzo, A. S. Coelho, D. Pellegrino, M. S. Mendes, L. Acioli, K. N. Cassemiro, D. Felinto, A. Zavatta, and M. Bellini, Phys. Rev. Lett. 116, 023602 (2016).

[3] C. Xiong, X. Zhang, Z. Liu, M. J. Collins, A. Mahendra, L. G. Helt, M. J. Steel, D.-Y. Choi, C. J. Chae, P. H. W. Leong, B. J. Eggleton, and B. Dayan, Nat. Commun. 7, 10853 (2015).

[4] M. Afzelius, N. Gisin, and H. de Riedmatten, Phys. Today 68, 42 (2015).

[5] D. G. England, K. A. G. Fisher, J.-P. W. MacLean, P. J. Bustard, R. Lausten, K. J. Resch, and B. J. Sussman, Phys. Rev. Lett. 114, 053602 (2015).

[6] H. Gorniaczyk, C. Tresp, J. Schmidt, H. Fedder, and S. Hofferberth, Phys. Rev. Lett. 113, 053601 (2014).

[7] Y. L. A. Rezus, S. G. Walt, R. Lettow, A. Renn, G. Zumofen, S. Götzinger, and V. Sandoghdar, Phys. Rev. Lett. 108, 093601 (2012).

[8] L. Garziano, V. Macrì, R. Stassi, O. DiStefano, F. Nori, and S. Savasta, Phys. Rev. Lett. 117, 043601 (2016).

[9] R. Loudon, The Quantum Theory of Light (Oxford University Press, Oxford, UK, 1983).

[10] Y. Silberberg, Annu. Rev. Phys. Chem. 60, 277 (2009).

[11] P. M. Kraus, B. Mignolet, D. Baykusheva, A. Rupenyan, L. Horný, E. F. Penka, G. Grassi, O. I. Tolstikhin, J. Schneider, F. Jensen, L. B. Madsen, A. D. Bandrauk, F. Remacle, and H. J. Wörner, Science 350, 790 (2015).

[12] K. Fisher, D. G. England, J. W. Philippe, W. Maclean, P. J. Bustard, K. J. Resch, and B. J. Sussman, Nat. Commun. 7, 11200 (2016).

[13] S. Kahra, G. Leschhorn, M. Kowalewski, A. Schiffrin, E. Bothschafter, W. Fuß, R. de Vivie-Riedle, R. Ernstorfer, F. Krausz, R. Kienberger, and T. Schaetz, Nat. Phys. 8, 238 (2012).

[14] A. M. Weiner, Rev. Sci. Instrum. 71, 1929 (2000).

[15] P. Brumer and M. Shapiro, Proc. Natl. Acad. Sci. USA 109, 19575 (2012).

[16] V. I. Prokhorenko, A. M. Nagy, S. A. Waschuk, L. S. Brown, R. R. Birge, and R. J. D. Miller, Science 313, 1257 (2006).

[17] A. Weigel, A. Sebesta, and P. Kukura, J. Phys. Chem. Lett. 6, 4032 (2015).

[18] G. Katz, M. A Ratner, and R. Kosloff, New J. Phys. 12, 015003 (2010).
[19] C.-C. Shu and N. E. Henriksen, J. Chem. Phys. 134, 164308 (2011).

[20] A. García-Vela and N. E. Henriksen, J. Phys. Chem. Lett. 6, 824 (2015).

[21] A. C. Han and M. Shapiro, Phys. Rev. Lett. 108, 183002 (2012).

[22] D. Meshulach and Y. Silberberg, Nature (London) 396, 239 (1998).

[23] N. Dudovich, B. Dayan, S. M. G. Faeder, and Y. Silberberg, Phys. Rev. Lett. 86, 47 (2001).

[24] A. Konar, V. V. Lozovoy, and M. Dantus, J. Phys. Chem. Lett. 3, 2458 (2012).

[25] J. P. Marangos, J. Mod. Opt. 45, 471 (1998).

[26] G. Heinze, C. Hubrich, and T. Halfmann, Phys. Rev. Lett. 111, 033601 (2013).

[27] A. V. Gorshkov, T. Calarco, M. D. Lukin, and A. S. Sørensen, Phys. Rev. A. 77, 043806 (2008).

[28] N. V. Vitanov, T. Halfmann, B. W. Shore, and K. Bergmann, Ann. Rev. Phys. Chem. 52, 763 (2001).

[29] K. Bergmann, N. V. Vitanov, and B. W. Shore, J. Chem. Phys. 142, 170901 (2015).

[30] M. Oberst, H. Münch, and T. Halfmann, Phys. Rev. Lett. 99, 173001 (2007).

[31] S. J. Glaser, U. Boscain, T. Calarco, C. P. Koch, W. Köckenberger, R. Kosloff, I. Kuprov, B. Luy, S. Schirmer, T. Schulte-Herbrüggen, D. Sugny, and F. K. Wilhelm, Eur. Phys. J. D 69, 279 (2015).

[32] J. Werschnik and E. K. U. Gross, J. Phys. B 40, R175 (2007).

[33] C. Brif, R. Chakrabarti, and H. Rabitz, New J. Phys. 12, 075008 (2010).

[34] C. M. Tesch and R. de Vivie-Riedle, Phys. Rev. Lett. 89, 157901 (2002).

[35] C.-C. Shu, T.-S. Ho, and H. Rabitz, Phys. Rev. A. 93, 053418 (2016).

[36] C.-C. Shu, T.-S. Ho, X. Xing, and H. Rabitz, Phys. Rev. A. 93, 033417 (2016).

[37] S. Zamith, J. Degert, S. Stock, B. de Beauvoir, V. Blanchet, M. A. Bouchene, and B. Girard, Phys. Rev. Lett. 87, 033001 (2001).

[38] D. Dong and I. R. Petersen, New J. Phys. 11, 105033 (2009).

[39] D. Daems, A. Ruschhaupt, D. Sugny, and S. Guérin, Phys. Rev. Lett. 111, 050404 (2013).

[40] S. Barbieri, P. Gellie, G. Santarelli, L. Ding, W. Maineult, C. Sirtori, R. Colombelli, H. Beere, and D. Ritchie, Nat. Photon. 4, 636 (2010). 